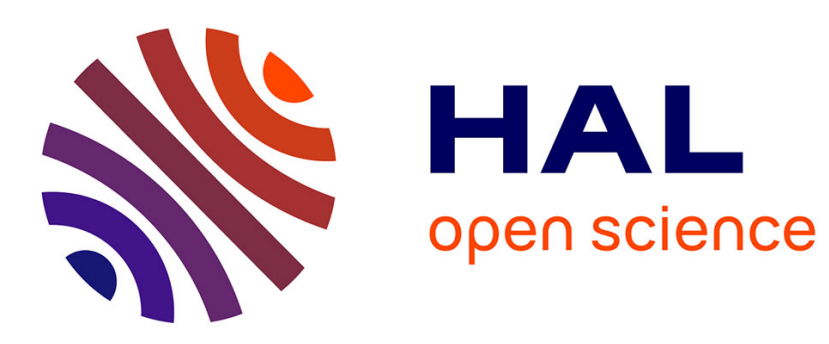

\title{
Optimal engagement and operation of a grid-connected PV/battery system
}

Arnold N'Goran, Bruno Daugrois, Marc Lotteau, Sophie Demassey

\section{To cite this version:}

Arnold N'Goran, Bruno Daugrois, Marc Lotteau, Sophie Demassey. Optimal engagement and operation of a grid-connected PV/battery system. IEEE PES Innovative Smart Grid Technologies Europe (ISGT-Europe 2019), Sep 2019, Bucharest, Romania. hal-02318181

\section{HAL Id: hal-02318181 \\ https: / hal-mines-paristech.archives-ouvertes.fr/hal-02318181}

Submitted on 16 Oct 2019

HAL is a multi-disciplinary open access archive for the deposit and dissemination of scientific research documents, whether they are published or not. The documents may come from teaching and research institutions in France or abroad, or from public or private research centers.
L'archive ouverte pluridisciplinaire HAL, est destinée au dépôt et à la diffusion de documents scientifiques de niveau recherche, publiés ou non, émanant des établissements d'enseignement et de recherche français ou étrangers, des laboratoires publics ou privés. 


\title{
Optimal engagement and operation of a grid-connected PV/battery system
}

\author{
Arnold N'Goran*, Bruno Daugrois ${ }^{\dagger}$, Marc Lotteau ${ }^{\dagger}$ and Sophie Demassey* \\ ${ }^{*}$ Centre for Applied Mathematics, MINES ParisTech, PSL Research University, \\ Sophia-Antipolis, France. Email: arnold.ngoran, sophie.demassey@mines-paristech.fr \\ ${ }^{\dagger}$ Bertin Technologies, Tarnos, France. Email: bruno.daugrois, marc.lotteau@bertin.fr
}

\begin{abstract}
Microgrid operation scheduling is a widely studied topic, but the performances of the solution algorithms proposed in the literature are rarely discussed and, in practice, scheduling is often only loosely optimized. In this paper, we present a practical context of coordination between a microgrid and an utility grid which concerns hundreds of microgrids worldwide. We adapt three distinct optimization strategies - mixed integer quadratic programming, simulation-based genetic algorithm and expertbased heuristic - and empirically compare their strengths. Our results emphasize the benefit of seeking for optimality, while the inaccuracies of the system dynamic model are compensated by periodic recomputation.
\end{abstract}

Index Terms-microgrids, optimal scheduling, mathematical programming, genetic algorithms, numerical simulation

\section{INTRODUCTION}

With the deregulation of power generation and the decarbonization targets set by countries worldwide, distributed generation with grid-connected renewable power plants are developing rapidly. Ranging from small solar rooftop systems to large power stations combining several sources, independent power systems feed power to the utility grid, ideally in a coordinated way: the independent producer seeks to maximize profit while respecting the constraints imposed by the grid operator to ensure network reliability and avoid voltage and frequency stability issues. Different coordination models exist to ensure equilibrium between the intermittent and unpredictable nature of renewable sources and the required stability of the power supply. A two-stage model is considered in the call for tenders CRE ZNI 2015 [1] launched by the French Energy Regulatory Commission to promote PV power generation facilities in small non-interconnected grids, mainly overseas islands. In this model, the producer first plans its injection/withdrawal, called its engagement profile, for the day after, by considering the solar irradiation forecasts. The producer must then ensure in real-time that the actual production meets the engagement to avoid high financial penalties. Storage devices, like lithium-ion battery banks, are needed to compensate forecast errors as well as PV unavailability periods to smooth the production and supply during the evening peak. Given a dynamic electricity tariff driven by the load forecast and the possibility to charge the batteries from the utility grid, the producer must then schedule the battery charge/discharge and injection/withdrawal operations over the day to maximize profit and minimize penalties. This involves solving two related optimization problems - to compute the engagement and to manage the production - which remain hard even with reasonable approximations in the system dynamic model.

Microgrid operation scheduling covers a large range of optimization problems, borrowing from economic dispatch, power flow and unit commitment, and their algorithmic solutions are widely studied (see e.g. recent surveys [2], [3]). The algorithms differ according to the specific features of the considered microgrids (e.g. on/off-grid, with/without storage or controllable sources) or the optimization paradigm, primarily chosen among mathematical programming (e.g. [4], [5]), dynamic programming (e.g. [6], [7]) or population-based heuristics such as particle swarm and genetic algorithms (e.g. [8][10]). Most of these studies focus on the operability of the microgrids, and do not discuss the choice of the algorithm, nor its performance in terms of runtime or optimality. Comparisons of different algorithms are even rarer, and mostly on variants of a same algorithm (see e.g. [11]), because simulating these algorithms in a real or realistic environment can be difficult to implement. A notable exception is the comparative study in [12] between a mathematical programming (MP) approach and a genetic algorithm (GA), but the experimental protocol is not detailed enough (how do the two models differ ? is MP solved at optimality ?) to explain the author's counterintuitive observation that the optimal MP solutions have lower profit than the approximated GA solutions. Finally, in practice, advanced optimization techniques are scarcely implemented and real microgrids are often managed with partial awareness of forecasts and optimality.

However, the financial gains of a microgrid rely on the scheduling algorithm and its ability to compute optimal or near-optimal solutions. This ability depends on the optimization paradigm, but is also correlated to the model granularity which impacts, in turn, the runtime performance of the algorithm. When scheduling is made in real-time, it is important to carefully choose the granularity as a trade-off between high profit solutions and low computation times.

In this paper, we study the real application context for microgrid scheduling specified in the CRE ZNI 2015 call for tenders [1], and which concerns about 150 winner projects of this and subsequent calls. This application is characterized by the two-phase engagement/control approach resulting in two related optimization problems, and by the penalty minimization criterion measured as a non-convex piecewise quadratic distance between two profiles. We then present a coarse-grain 
analytic formulation of the scheduling problems, as MixedInteger Quadratic Programs, and conduct experiments, on a real study-case which is representative of the CRE ZNI contractors. We compare mathematical programming (MP) with two distinctive approaches: a business-as-usual expertbased strategy (BAU) and a genetic algorithm (GA) embedding a fine-grain numerical simulator of the dynamical system. A key finding of these experiments is that, compared to the BAU strategy, the future-aware algorithms achieve substantial savings, ranging from 50 to 100 euros a day, corresponding approximately to $10 \%$ of the daily profits. This result, while predictable, should encourage microgrid operators to invest in optimization-oriented controllers. We also found that the MP solutions are always more profitable, while computed 70 times faster, than the simulation-based GA solutions. This suggests that it is preferable to optimize exactly over a rough model of the system dynamics than to optimize heuristically over a precise model. The main reason is that model inaccuracies are compensated in our experiments by the periodic recomputation of the production schedule in real-time control.

The paper is organized as follows: Section II defines the architecture of the microgrid and of the management workflow. Section III describes the three distinct optimization approaches. Section IV presents the experimental protocol, results and analysis.

\section{MicROGRID MANAGEMENT}

We illustrate the CRE ZNI 2015 coordination problem on a $1000 \mathrm{kWp} \mathrm{PV/battery} \mathrm{power} \mathrm{plant} \mathrm{installed} \mathrm{on} \mathrm{a} \mathrm{Carribean}$ island in response to the call for tenders, the same we used in our experimental protocol.

\section{A. The microgrid and controller architectures}

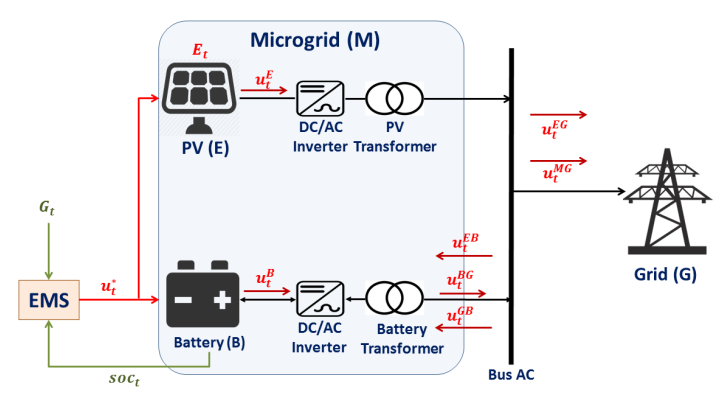

Fig. 1. Architecture of the microgrid

The microgrid illustrated in Figure 1 is made of a PV array (E) and a battery bank (B) equipped with power converters and transformers and is connected to the public distribution power grid $(\mathrm{G})$ of the island. The power flows through the microgrid and from/to the grid are depicted by red arrows. The microgrid is operated by a Model Predictive Control (MPC) implementing an Energy Management System (EMS) coupled with a Power Management System (PMS). They are made of precisely three components run at different frequencies on different discretized time horizons, each denoted as a sequence of time steps $t \in\{1, \ldots, T\}$. The Predictor determines the engagement profile $d \in \mathbb{R}^{T}$ for the day after with a sampling time of 1 minute (i.e. $T=1440$ ), given: unit electricity tariff profile $\rho \in \mathbb{R}_{+}^{T}$, PV generation forecast profile $E \in \mathbb{R}_{+}^{T}$, and the current state $x_{0}$ of the system (including the battery state of charge). Engagement $d_{t}$ is defined as the stationary electric power during time step $t$, that is injected to the grid if positive, or drawn from the grid if negative. The predictor is run once at midnight, and possibly every 6 hours with updated forecasts. The engagement is sent to the grid operator and as input to the Tracker. The Tracker computes every 15 minutes, at time $t$, the production (i.e. injection or withdrawal) plan $u \in \mathbb{R}^{T-t}$ until the end of the day with a sampling time of 1 minute, using the observed plant state $x_{t}$ and updated forecasts $E \in \mathbb{R}_{+}^{T-t}$. The $P M S$ attempts to apply the Tracker command $u_{t}$ for every minute $t$. It is in charge of the dispatch and communicates every second with the plant by observing the current state and PV generation and returning the real time control for the next second. The PMS relies on a rule-based system which allows to change command $u_{t}$ when it is not applicable.

\section{B. The coordination model}

The CRE ZNI 2015 coordination model [1] is characterized by (1) an incentive for injection in the evening in the form of higher electricity prices $\rho_{t}$ during a fixed 2-hour peak period, and (2) financial penalties $C_{t}^{P}$ incurred at any time $t$ when production $u_{t}$ does not respect engagement $d_{t}$ within a tolerance range of $5 \%$ of the installed power $P_{\text {inst }}$. Penalties are computed as follows. Let $d^{-}=d-0.05 P_{\text {inst }}$ and $d^{+}=d+0.05 P_{\text {inst }}$ denote the tolerance limits, then $C_{t}^{P}=0$ when the engagement is satisfied $\left(d_{t}^{-} \leq u_{t} \leq d_{t}^{+}\right)$, $C_{t}^{P}=\rho_{t} u_{t}$ in case of overproduction $\left(u_{t}>d_{t}^{+}\right)$, and $C_{t}^{P}=\rho_{t} \Delta\left(u_{t}, d_{t}\right)$ in case of underproduction $\left(u_{t} \leq d_{t}^{-}\right)$, with $\Delta(u, d)=\frac{\left(u-d^{+}\right)^{2}-\left(d^{-}-d^{+}\right)^{2}}{P_{\text {inst }}}$. The producer's benefit at time $t$ includes electricity sale and purchase and is thus equal to $J_{t}\left(u_{t}\right)=\rho_{t} u_{t}-C_{t}^{P}$. Note that, for a given engagement value $d_{t}, J_{t}$ is a non-concave piecewise quadratic function.

The shape of the engagement profile $d$ is defined by the grid operator as specified bounds on the ramp rates $d_{t+1}-d_{t} \in$ $\left[\alpha_{t}^{-}, \alpha_{t}^{+}\right]$or on the power $d_{t} \in\left[\beta_{t}^{-}, \beta_{t}^{+}\right]$(see values in [1]). These bounds primarily differ during the evening peak period.

\section{OPTIMIZATION STRATEGIES}

\section{A. Mixed Integer Quadratic Programming (MP)}

Linear Programming is widely used for microgrid operation scheduling for its two main benefits: (1) by using efficient off-the-shelf solvers, it requires almost no algorithmic development, and (2) it converges to optimal solutions and provides optimality certificates. In return, modelling the system dynamics (the battery operation, for example) as linear constraints involves deep simplifications. Introducing discrete variables does not help to improve the physical model accuracy but it allows to handle logical constraints and binary states. Despite the higher algorithmic complexity, discrete models of limited size can efficiently be optimized by the state-of-the-art solvers.

We adapt the standard mathematical programming formulation of the production scheduling problem to our context. 
The decision variables are the power flows $u$ between PV $(P)$, battery $(B)$ and grid $(D)$ depicted in Figure 1 and the battery state of charge $s o c$ (with initial $s o c_{0}$ fixed to the currently observed state). They are related by the usual balance constraints (2), (3). PV curtailment is allowed by (4). The efficiency of inverters and transformers are explicit in this model through coefficients $\eta$ - as well as additional equipment losses $u^{L}(5)$. Two vectors of binary variables are required here: by (7)-(8), $y_{t}^{B}$ distinguishes battery charging from discharging, as the associated efficiencies $\eta_{c}$ and $\eta_{d}$ are different, given $\beta_{c}^{+}$ and $\beta_{d}^{+}$the maximum charge and discharge values; by (9)(10), $y_{t}^{G}$ distinguishes injection (then $u_{t}=u_{t}^{M G} \geq 0$ ) from withdrawal (then $u_{t}=-u_{t}^{G B} \leq 0$ ). However, no additional binary variables are required to model the piecewise objective functions $J_{t}$ in this context, observing that overproduction is non-optimal as curtailment is allowed (thus (9) enforces $\left.u_{t} \leq d_{t}^{+}\right)$, and that $J_{t}\left(u_{t}\right)=\rho_{t} \max \left(u_{t}, u_{t}-\Delta\left(u_{t}, d_{t}\right)\right)$ for any $u_{t} \leq d_{t}^{+}$(which is enforced by (11) and (12) given that $u=u^{\bar{M} G}-u^{G B}$ and $\left.z=J / \rho\right)$. Finally, Constraints (12) being concave quadratic, modern Mixed Integer Quadratic Programming solvers can directly solve this model.

$$
\begin{array}{lc}
\max \sum_{t} \delta_{t} \rho_{t} z_{t} & s . t .: \\
\frac{s o c_{t}-s o c_{t-1}}{\delta_{t}}=\eta_{c}\left(u_{t}^{E B}+u_{t}^{G B}\right)-\frac{u_{t}^{B G}}{\eta_{d}} & \forall t \\
u_{t}^{E G}+\eta_{b t} u_{t}^{B G}-u_{t}^{L}=u_{t}^{M G} & \forall t \\
u_{t}^{E B}+u_{t}^{E G} \leq \eta_{p t} E_{t} & \forall t \\
u_{t}^{L} \geq \frac{1-\eta_{b t}}{\eta_{L}}\left(u_{t}^{E B}+u_{t}^{G B}+u_{t}^{B G}\right) & \forall t \\
y_{t}^{G} \leq y_{t}^{B}, y_{t}^{G} \in\{0,1\}, y_{t}^{B} \in\{0,1\} & \forall t \\
0 \leq u_{t}^{B G} \leq \beta_{d}^{+} y_{t}^{B} & \forall t \\
0 \leq u_{t}^{E B}+u_{t}^{G B} \leq \beta_{c}^{+}\left(1-y_{t}^{B}\right) & \forall t \\
0 \leq u_{t}^{M G} \leq d_{t}^{+} y_{t}^{G} & \forall t \\
0 \leq u_{t}^{G B} \leq \beta_{t}^{-}\left(y_{t}^{G}-1\right) & \forall t \\
z_{t} \leq u_{t}^{M G}-u_{t}^{G B} & \forall t \\
z_{t} \leq u_{t}^{M G}-u_{t}^{G B}-\Delta\left(u_{t}^{M G}-u_{t}^{G B}, d_{t}\right) & \forall t \\
0 \leq s o c_{t} \leq 1 & \forall t
\end{array}
$$

We optimize the engagement using the same model after setting vector $d$ as decision variables and adding the ramp and power bounds $\alpha$ and $\beta$ (see Section II-B) as linear constraints.

\section{B. Simulation-Based Genetic Algorithm (GA)}

A numerical software is best suited to accurately model dynamical systems like microgrids, but it would only simulate one production plan at a time. To be used in microgrid scheduling, it must be combined with an optimization strategy, guided by an estimate of the profit to generate the sequence of production plans to evaluate by the simulator. Genetic Algorithm (GA) is a favorite strategy: (1) the global performance of this metaheuristic is widely recognised, (2) it is population-based and thus can easily be interfaced to a blackbox simulator to evaluate each individual, (3) generic configurable implementations exist. Contrarily to the MP approach, however, the convergence to an optimum is not guaranteed and it does not compute upper bounds on the objective to estimate the quality of the returned solutions.

In our implementation on the production control problem, individuals are potential production plans $u \in \mathbb{R}_{+}^{T}$. During the optimization, a population of POPSIZE individuals is generated. At the first iteration, individuals are sampled from a problem knowledge-based production plan, following a Gaussian distribution. Iteratively, new individuals are generated by selection, combination and mutation of the individuals of the previous generation. At this step, only the bounds on the production variables are enforced to assert the feasibility of the individuals. Each new generated individual is then evaluated by the simulator to assert its feasibility regarding the physical constraints, then the actual profit of the individual is computed. Individuals with higher profits are used in priority to generate new individuals, while low profit individuals may be discarded from the population. After $N G E N$ iterations, an individual with the maximum profit is returned. We use the same algorithm to optimize the engagement, by just ignoring penalties when computing the individual profits.

\section{Expert system (BAU)}

The third strategy is representative of expert microgrid management policies which are common in practice. From the CRE ZNI 2015 specifications and the specified ramp bounds, we can draw the general shape of a valid production profile as depicted in Figure 2.

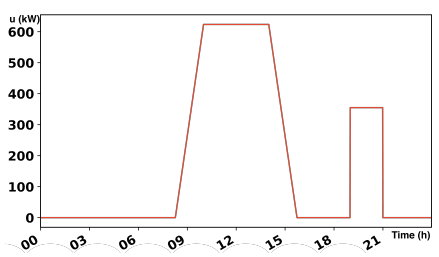

Fig. 2. A valid production profile for the CRE ZNI 2015 model.

The main policy is to fully charge the battery when sun is available (typically between 6AM and 6PM) in order to satisfy the peak constraint and get the maximum profit during the peak period (between 7PM and 9PM). After having charged the battery, the remaining solar production is used to directly supply the grid. Using this policy and the knowledge of the maximum power slope and of the plateau phase start time (10AM) and end time (2PM), simple arithmetic calculations are used to derive values for the trapezoid slopes and for the peak height, thus to fully determine the engagement profile $d$ for the day. With this strategy, the production control $u$ is set to $d+0.05 P_{\text {inst }}$ during peak period and matches engagement $d$, elsewhere. 


\section{BENCHMARK ANALYSIS}

\section{A. Experimental settings}

Our benchmark set is built on the real power plant described in Section II with $P_{\text {inst }}=1000 \mathrm{~kW}$ and a battery nominal capacity of $1000 \mathrm{kWh}$. For a fair comparison between the different optimization solutions, we use historical solar irradiation data in place of forecasts to erase stochasticity. Hence we built a benchmark of 24 instances by considering the irradiation profiles observed at the plant location for 2 random days each month of year 2015. Daily profiles $E$ are given from midnight to midnight with a discretization step of one minute.

Because experiments on sampling data cannot be run on the real plant, we simulate the system with a numerical model implemented with MATLAB/Simulink instead. This reference model has empirically shown to be very accurate. The PMS thus commands this model in place of the plant. The same model is implemented as the black-box solver in the simulation-based GA.

Experiments were performed on an Intel Core i7-6700HQ OctaCore computer at $2.6 \mathrm{GHz}$ and $16 \mathrm{Go}$ RAM. The genetic algorithm was implemented using the Python library DEAP 1.0.2 and the mathematical model solved with the Gurobi 7.5.2 MIQCP solver using the default parameter set. The BAU strategy is implemented in Python 3.5.

\section{B. Evaluating the modeling flaws}

Our first experiment evaluates the accuracy of the MP model regarding the reference model. For that purpose, we implemented the MP model within the Tracker to optimize the production plans, and compared the conceptual optimal solution $(u, s o c, J)$ of MP with the "real" solution of the reference model, observed after applying control $u$ through the PMS. In the standard setting, denoted P15, the Tracker is run every 15 minutes and the updated production control applied for the next 15 minutes. The conceptual solution is thus recomposed by concatenating the sequence of 15-minutes profiles. To amplify the impact of the MP model approximation, we also considered a fictional setting PP where the optimal production control is computed only once at midnight and applied the entire day. We also evaluated a simpler MP model where the equipment losses are neglected, by relaxing Constraints (5) and fixing the inverter and transformer efficiencies to 1 . The settings corresponding to the two execution modes above are denoted N15 and PN, respectively.

Table I reports, for the 4 settings, the deviation between the MP conceptual solution and the observed result, evaluated through 3 numerical indicators computed in average on the 24 instances (avg) and for the worst case (max): the difference of the daily profits $(\Delta J)$ and the Mean Absolute Error ${ }^{1}$ on the battery state of charge $(\Delta s o c)$ and on the command $(\Delta u)$. The last two columns $(J)$ show the observed generated profit in average (avg) and the standard deviation (stddev).

We first observe that the deviations on the battery state of charge and on the production control are limited even for

\footnotetext{
${ }^{1}$ for two time series $u, v \in \mathbb{R}^{T}, M A E(u, v)=\frac{1}{T} \sum_{t}\left|u_{t}-v_{t}\right|$.
}

TABLE I

DEVIATION BETWEEN THE MP OPTIMAL SOLUTION AND THE RESULT OF THE OPTIMAL PRODUCTION CONTROL, AND GENERATED PROFITS.

\begin{tabular}{l|rr|rr|rr||rr}
\hline & \multicolumn{2}{|c|}{$\Delta J(€)$} & \multicolumn{2}{c|}{$\Delta s o c(\%)$} & \multicolumn{2}{c|}{$\Delta u(\mathrm{~kW})$} & \multicolumn{2}{|c}{$J(€)$} \\
& avg & $\max$ & avg & $\max$ & avg & $\max$ & avg & stddev \\
\cline { 2 - 8 } PP & 0.3 & 1.4 & 3.33 & 3.92 & 0.08 & 0.13 & 964.3 & 156.7 \\
P15 & 0.8 & 5.9 & 0.11 & 0.147 & 0.06 & 0.14 & 1006.3 & 158.0 \\
PN & 61.6 & 81.1 & 5.18 & 6.54 & 4.56 & 5.96 & 970.9 & 152.5 \\
N15 & 0.0 & 0.2 & 0.09 & 0.11 & 0.06 & 0.09 & 1009.3 & 160.3 \\
\hline
\end{tabular}

the worst-case PN. The approximations of the MP model are thus reasonably accurate in this context. The deviations are negligible when MP is solved repeatedly every 15 minutes (for P15 and N15) since, each time, the production plan is readjusted according to the real state of the plant at the beginning of the period. This shows how the periodic run of the Tracker makes the overall management procedure robust to the errors on the physical model in the Tracker. Incidentally, the smallest average deviations are achieved by N15 when equipment losses are fully neglected, although the model in P15 seems more realistic. Indeed, both models are approximations, and one is not theoretically better than the other. When observing the generated profits $J, \mathrm{P} 15$ and N15 achieve the highest profits. This is not surprising, since the MP solutions were always optimal (for the conceptual model), thus, given they are nearly feasible in P15 and N15, they are probably close to the real feasible optima.

Figure 3 illustrates the impact of the model approximation in setting PP on the day where we observed the highest deviation. Figure on the left depicts the battery state of charge in the optimal MP solution $s_{0}{ }^{o p t}$ (in red) and the observed profile $s o c^{r e a l}$ (in blue). Figure on the right depicts the production plan $u$ in the MP optimal solution $u^{\text {opt }}$ (in red) and the observed profile $u^{\text {real }}$ (in blue). The engagement $d$ is depicted in purple and the tolerance range in green.
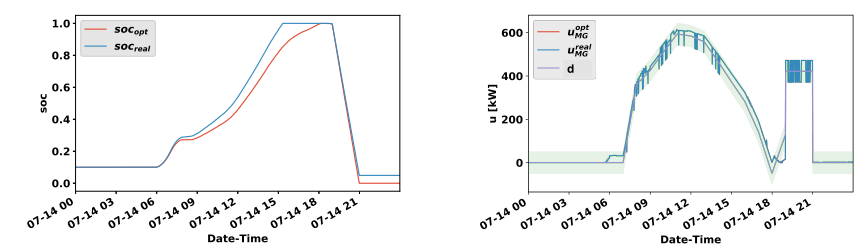

Fig. 3. Profiles of state of charge (left) and production plan (right) in the MP optimal solution (red) and as observed (blue) for setting PP.

The state of charge is under-estimated in the optimal MP solution (on the left in red) and the battery actually reaches it maximal capacity more than 1 hour before than expected. However the error does not exceed $12 \%$ of the battery capacity at any time. Observing the output power $u$ (on the right), the absolute error oscillates between 0 and $1 \mathrm{~kW}$, and is thus clearly inside the tolerance range $[-50 \mathrm{~kW}, 50 \mathrm{~kW}]$.

Finally, we also experimented with a higher recomputation frequency, from 15 to 5 minutes, but we did not observe a significant improvement of the results. 


\section{Comparing the optimization techniques}

Our second experiment compares the solutions of MP and $\mathrm{GA}$, in order to evaluate the benefits of a finer model (using the simulation-based GA) or of a better guided optimization strategy (using MP). Two implementations of the Tracker, with MP and with GA, are provided. For a fair comparison, we first use the same engagement profile, obtained with GA. The MP Tracker is run in the standard setting P15, i.e. updating the production control every 15 minutes. We evaluate the solution in the reference model after applying the control through the PMS. Because, GA solutions are feasible for the reference model, since it uses the same simulator, it is not required to update the solution periodically, thus GA is run only once at midnight as the engagement. The algorithm parameters (maximum 10 generations and 400 individuals per generation) were chosen empirically as the best balance between low solution time and high profit.

First, the runtime of MP Tracker is about 3.6s in average with a standard deviation of $3.3 \mathrm{~s}$, while the runtime of GA Tracker is about $251 \mathrm{~s}$ with a standard deviation of $15.2 \mathrm{~s}$. The MP Tracker is then about 70 times faster than GA Tracker, but both are eligible for a use on site since 15 minutes are allowed to the computation.

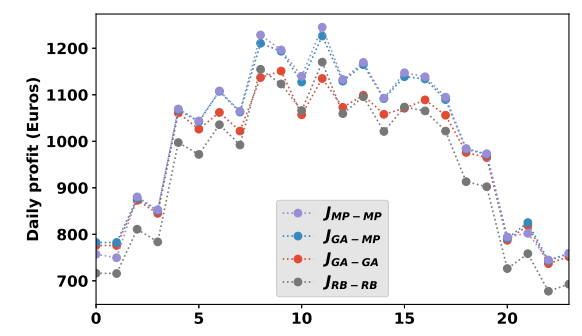

Fig. 4. Daily profit over the 24 days.

Comparing the generated profits over the 24 instances, we see on Figure 4 that the MP Tracker $\left(J_{G A-M P}\right.$ in blue) is always more profitable than the GA Tracker $\left(J_{G A-G A}\right.$ in red) up to $9.7 \%$ higher (about $110 €$ ). The average daily profits are, respectively, 1006 and $975 €$, with a standard deviation of 156 and $136 €$. The difference is significant in practice and it confirms our first analysis that a detailed model of the system dynamics is not a requirement. This also highlights the benefit of solving the problem at optimality since GA did not generate the individuals correponding to the production plans obtained with MP.

Finally, we observe on Figure 4, that higher profits were obtained for 21 instances out of 24 when computing the engagement, in the Predictor, also with an MP solver (see configuration $J_{M P-M P}$, in purple), but the difference with $J_{G A-M P}$ is tight.

\section{Worth of optimization}

Finally, our third experiment evaluates the worth of applying advanced optimization techniques compared to simplest BAU strategies. We thus experimented the BAU strategy described in Section III-C and observed the real production achieved when applying this command through PMS. The generated profits are depicted as $J_{R B-R B}$ (in grey) on Figure 4.

BAU generate profits slightly higher that GA on only 3 instances, where MP was significantly higher. On the 21 other instances, GA and MP profits were up to 100 euros higher.

\section{CONClusion}

On our study case, the MP-based approach constantly generated the highest profits - often significantly higher than the two alternatives - in only few seconds of computation time. It suggests that seeking for optimality is profitable, even when considering a loose approximation of the system dynamics, thanks in particular to the periodic recomputation of the solutions. Because one main criticism of the MP approach is to rely on an expensive proprietary solver, we should note that open-source solvers could be used instead to compute optimal solutions in much less time than the 15 minutes allowed. Finally, because mathematical programming is flexible enough to handle model variants and uncertain data, it could be interesting to have the confirmation of these observations on various microgrid study cases and, in the opposite case, to understand the limits of the approach.

\section{REFERENCES}

[1] Commission de Régulation de l'Energie (CRE - France), “Appel d'offres portant sur la réalisation et l'exploitation d'installations de production d'électricité à partir de techniques de conversion du rayonnement solaire d'une puissance supérieure à $100 \mathrm{kwc}$ et situées dans les zones non interconnectées," 2015, [Online; accessed 22-December-2018].

[2] N. Karthik, A. K. Parvathy, and R. Arul, "Optimal operation of microgrids-a survey," International Journal of Applied Power Engineering, vol. 7, no. 2, pp. $179-185,2018$.

[3] L. I. Minchala-Avila, L. E. Garza-Castanon, A. Vargas-Martinez, and Y. Zhang, "A review of optimal control techniques applied to the energy management and control of microgrids," Procedia Computer Science, vol. 52, pp. $780-787,2015$.

[4] R. Palma-Behnke, C. Benavides, F. Lanas, B. Severino, L. Reyes, J. Llanos, and D. Saez, "A microgrid energy management system based on the rolling horizon strategy," IEEE Transactions on Smart Grid, vol. 4, no. 2, pp. 996 - 1006, 2013.

[5] P. Malysz, S. Sirouspour, and A. Emadi, "Milp-based rolling horizon control for microgrids with battery storage," 39th annual conf. of the IEEE IES (IECON 2013), pp. 2099 - 2104, 2013.

[6] A. Das, Z. Ni, and X. Zhong, "Near optimal control for microgrid energy systems considering battery lifetime characteristics," IEEE Symposium Series on Computational Intelligence (SSCI), 2016.

[7] S. K. Jadhav, "Optimal power flow in wind farm microgrid using dynamic programming," Int. Conf. on Emerging Trends and Innovations In Engineering And Technological Research (ICETIETR), 2018.

[8] C. Chen, S. Duan, T. Cai, B. Liu, and G. Hu, "Smart energy management system for optimal microgrid economic operation," IET Renewable Power Generation, vol. 5, no. 3, pp. 258 - 267, 2011.

[9] A. Askarzadeh, "A memory-based genetic algorithm for optimization of power generation in a microgrid," IEEE Transactions on Sustainable Energy, vol. 9, no. 3, pp. 1081 - 1089, 2018.

[10] M. Nemati, M. Braun, and S. Tenbohlen, "A memory-based genetic algorithm for optimization of power generation in a microgrid," Applied Energy, vol. 210, pp. 944 - 963, 2018.

[11] A. Maulik and D. Das, "Optimal operation of microgrid using four different optimization techniques," Sustainable Energy Technologies and Assessments, vol. 21, pp. 100-120, 2017.

[12] C. Hu, S. Luo, Z. Li, X. Wang, and L. Sunr, "Energy coordinative optimization of wind-storage-load microgrids based on short-term prediction," Energies 2, vol. 8, pp. 1505 - 1528, 2015. 